\title{
Current problems in microbiology: 1 Chlamydial infections: Which laboratory test?
}

\author{
G L Ridgway, D Taylor-Robinson
}

Introduction

Chlamydial infections are now recognised as being common. Trachoma, caused by Chlamydia trachomatis, remains a major cause of blindness in the third world. Other serovars of this species are responsible for nonblinding paratrachoma, for lymphogranuloma venereum, and for genital infections in men and women. Ascending infection of the female genital tract may result in cervicitis, endometritis, or salpingitis, with an increased risk of infertility or ectopic pregnancy. Infection of the fetus may cause conjunctivitis, otitis media, pharyngitis or pneumonitis. Another complication is sexually acquired reactive arthritis, including Reiter's syndrome. ${ }^{1}$ Human $C$ psittaci infection typically affects the respiratory tract, producing an atypical pneumonia syndrome, but may occasionally manifest as a culture negative endocarditis. Organisms within this species are of profound economic importance in veterinary medicine, because they particularly affect birds and sheep. $C$ pneumoniae, a third species, has now been described; this may prove an important cause of human to human respiratory infection. ${ }^{2}$

The need for a routine diagnostic service for chlamydial infections is clear, but meeting it may be extremely difficult. Although chlamydiae are bacteria, they cannot be cultivated in non-living or cell free media. Their isolation requires the use of cell cultures, but in the United Kingdom such facilities are restricted essentially to virus laboratories, which, from a diagnostic service point of view, are often organised on a regional basis. Furthermore, cell culture procedures are expensive and labour intensive. The use of non-culture techniques is therefore very attractive.

Department of

Clinical Microbiology,

University College

Hospital, London

WCiE 6AU

G L Ridgway

Clinical Research

Centre, Northwick

Park Hospital,

Harrow, Middlesex

HA1 3UJ

D Taylor-Robinson

Correspondence to:

Dr G L Ridgway

Accepted for publication 5 July 1990 means. Swabs from the male urethra should be obtained by passing a cotton-wool tipped swab 3-4 cm down, and rotation before removal. ${ }^{3}$ The cervix should be wiped clean before sampling the squamo-columnar junc- tion with a cotton-wool tipped swab. Specimens from the eyes of patients with trachoma are obtained by gently scraping the upper tarsal conjunctiva; for the diagnosis of paratrachoma or chlamydial ophthalmia neonatorum, the lower tarsal conjunctiva should be firmly swabbed. Mahony and Chernesky found that cotton swabs were superior to those of calcium alginate, and that aluminium shafted swabs were superior to plastic for the isolation of $C$ trachomatis. ${ }^{4}$ Swabs on wood or Dacron performed poorly. They also warned that swabs provided commercially for enzyme immunoassay (EIA) tests may be toxic if used to collect specimens for culture. Furthermore, Dacron swabs may produce artefacts in cell cultures stained with iodine, while alginate swabs may produce non-specific fluorescence in direct immunofluorescence tests or on dark ground microscopy. ${ }^{5}$ An alternative method for collecting specimens from the cervix is to use a brush. Moncada et al compared the use of a brush with alginate or Dacron swabs for collecting specimens for culture or direct immunofluorescence, and found that the brush improved significantly the sensitivity of both methods. ${ }^{6}$ Taylor-Robinson, ${ }^{7}$ however, commenting on the finding by Ciotti, Sondheimer, and Nachamkin ${ }^{8}$ that the use of a brush had increased the detection of $C$ trachomatis from $12 \%$ to $27 \%$ in a low risk population, warns of the possibility of an increase in false positive direct immunofluorescence results owing to non-specific fluorescence by the products of blood induced and collected by the brush.

For direct immunofluorescence examination, swabs are rolled or rubbed directly on a glass slide, and other specimens are smeared. Other methods (culture, EIA) require the use of a suitable transport medium. For culture, 2-sucrose phosphate supplemented with fetal calf serum is preferred ${ }^{5}$ while for commercial EIA methods, the transport buffer supplied by the manufacturer must be used. Temperature of storage will strongly affect the survival of chlamydiae. Storage of specimens at $4^{\circ} \mathrm{C}$ for more than 24 hours reduces viability, while freezing at $-70^{\circ} \mathrm{C}$ (but not $-20^{\circ} \mathrm{C}$ ) will preserve viability, albeit with some reduction in the number of viable organisms. ${ }^{4}$ Further studies on the effect of storage on EIA results are required, but it seems reasonable to assume that the temperature of storage would 
be less critical than it would be for preserving viability. The repeated sampling of a single site, or the sampling of multiple sites, has been recommended by some workers, ${ }^{910}$ although this may lead to an unacceptable increase in cost without a substantial increase in the yield of isolates. ${ }^{11}$ The pooling of samples from the urethra and cervix in women may be an acceptable alternative. ${ }^{12}$

\section{Cell culture}

Many cell lines are suitable for the culture of chlamydiae, and the subject has been reviewed recently by Barnes. ${ }^{5}$ The method considered by many to be optimal involves the use of McCoy cells treated with cycloheximide, with one blind passage, and staining of the cell monolayer with a monoclonal immunofluorescent antibody. This procedure will be expensive, both in material and in technical time, although relative costs will be reduced when large numbers of specimens are being processed. The delay brought about by the addition of a blind passage may be clinically unacceptable, particularly with neonatal specimens. Cell culture is still regarded by many as the method of choice for chlamydial diagnosis probably because, in competent hands, there can be little doubt that an isolate does indeed belong to the Chlamydia genus. The method is far from perfect, however, as estimates suggest, for example, that the sensitivity of culture of a single cervical specimen is between $75 \%$ and $80 \%$ when compared with direct immunofluorescence and EIA combined. ${ }^{13}$ Furthermore, the method is fraught with the difficulties inherent in handling cell cultures, destruction of cells by some specimens being one. The high specificity of the cell culture technique described above, however, is the reason why it is often used as the "gold" standard against which other non-culture methods are compared. Nevertheless, in view of the failings of cell culture, it is easy to understand why this stance is ignored by some who, in view of their expertise, feel that they can place greater reliance on direct immunofluorescence as the comparator.

\section{Evaluation of new tests}

When a new test is compared with a standard method, it is customary to express the results in terms of sensitivity, specificity, predictive value of a positive result (PVP), and predictive value of a negative result (PVN). It is important to appreciate that the PVP and PVN depend on the prevalence of infection in the population under investigation. Thus for a test with a sensitivity of $87 \%$ and a specificity of $98 \%$, the PVP will be $81 \%$ for a prevalence of $16.8 \%$ for example, STD clinical attenders-but only $50 \%$ for a prevalence of $2.5 \%$-for example, family planning clinic attenders. This means that the use of antigen detection tests in low risk populations is liable to produce proportionately more false positive results than in high risk groups. Obviously, this is particularly undesirable when dealing with a sexually trans- mitted infection. To this problem must be added the consequences of not detecting all infections by the comparative cell culture procedure, as well as the difficulty of knowing whether a "standard" method behaves comparably in different centres, and the inherent variability of collection and transport systems. Attempts have been made to resolve discrepancies by comparing two or more antigen tests (including the new test) with cell culture. ${ }^{14}$ There is no path that avoids all the problems, however, and the laboratory scientist and the clinician must be aware of the shortcomings of diagnostic tests if embarrassing misdiagnoses are to be avoided; clinicians should not fall into the trap of trying to fit the results to their clinical impression. The laboratory can be wrong and unexpected positive results should always be confirmed by additional tests, or by repeating the investigation using a highly specific method, such as cell culture.

\section{Direct antigen detection}

The direct examination of clinical material for chlamydiae is not new. Indeed, for over $\mathbf{5 0}$ years, until the advent of culture it was the only method available. Staining clinical material by either Giemsa reagent or iodine is insensitive and not very specific, however, and can no longer be recommended. Furthermore, the use of Papanicolaou staining for this purpose is to be discouraged. Forster et al found that only $13 \%$ of women with positive cultures had Papanicolaou stain findings suggestive of chlamydial infection, and only $40 \%$ of women with smear findings indicating infection yielded $C$ trachomatis on culture. ${ }^{15}$ Direct antigen detection tests will eliminate the problem of having to maintain survival during transportation, but they cannot compensate for poor quality specimens. The discipline of following the manufacturer's protocol for performing these tests ought to remove much of the variability of results among centres, yet the results of reported clinical trials show that this ideal is also some way off.

\section{Direct immunofluorescence}

In 1982 Tam et al reported that a specific monoclonal antibody against the outer membrane protein (MOMP) could be used in a direct immunofluorescence test to detect elementary bodies in clinical material. ${ }^{16}$ Thomas et al evaluated direct immunofluorescence staining by comparing it with fluorescent antibody or Giemsa stained cell cultures. ${ }^{17}$ Agreement between the results of direct immunofluorescence and those of cell culture was very good, being $100 \%$ for female contacts of men with non-gonococcal urethritis (NGU), $99 \%$ for men with NGU, and $94 \%$ for men with gonorrhoea. Agreement was less impressive when the recommended criterion of 10 or more elementary bodies was used to score a result as positive. Of course, this does raise the question of what number should be used. The choice of cut off value must depend on the manufacturer's recommendations, the 
experience of the laboratory (some investigators being confident of using less than 10 elementary bodies, even one), and the results from other centres using the same test on similar populations. ${ }^{5}$ It must be appreciated that for the inexperienced any improvement in sensitivity may be at the expense of specificity, and with a reduction in the PVP. Certainly, the subjectivity of direct immunofluorescence is a cause for concern. False positive results will occur, and the comment by Jones and TaylorRobinson that the widespread use of direct immunofluorescence staining would result in a rise of cases falsely attributable to chlamydiae has proved salutary. ${ }^{18}$ Direct immunofluorescence, however, is the only method which allows the adequacy of the specimen to be evaluated, and it is convenient for handling small numbers of specimens, or where a rapid result is required.

The commercially available direct immunofluorescence monoclonal antibodies are directed against either the species specific MOMP, or the genus specific lipopolysaccharide (LPS). Thus while the former will detect only $C$ trachomatis, the latter will detect all three species theoretically, but will be unable to distinguish between them. With regard to the diagnosis of oculo-genital infections this is of little consequence as the only expected chlamydial species is $C$ trachomatis. When direct immunofluorescence tests are being used on specimens from other sites, however, particularly where validation studies have not been performed, caution is required before drawing conclusions as to the nature of the infectionnot only as to whether it is chlamydial, but also as to which species the organism belongs.

\section{Enzyme immunoassay (EIA)}

EIA techniques offer the potential advantages over direct immunofluorescence of automation and lack of subjectivity. They suffer, however, from the basic drawback of a grey zone existing between definite positive readings of optical density and definite negative readings. The "clinical" cut off (usually determined by the manufacturer's recommendation) will be designed to reduce as far as possible the likelihood of false positive results-that is, maintaining specificity-but this will of necessity be at the expense of sensitivity.

All currently available EIA tests utilise either polyclonal or monoclonal antibodies directed predominantly against chlamydial LPS. They are, therefore, all genus specific. Mumtaz et al obtained a sensitivity of $97 \%$ and a specificity of $92.5 \%$ in a comparison of Chlamydiazyme (Abbott Laboratories) with single cycle cell culture ${ }^{19}$; results that compare favourably with direct immunofluorescence methods. Others have been less enthusiastic. ${ }^{20}$ In a comparative study of IDEIA (Novo-Bio Laboratories) and direct immunofluorescence, Thomas et al found that pooling three specimens from the cervix improved the sensitivity of IDEIA from 74 to $96 \%$, while maintaining specificity at $97 \% .{ }^{21}$ False positive results occur with EIA methods, often caused by cross reactivity with other bacterial species. ${ }^{22}$ It is therefore desirable, although not always done, to confirm positive results and to evaluate further, borderline ones. This may be accomplished by transferring the remaining EIA transport medium to a slide (either in total, or after centrifugation) and testing with a MOMP specific immunofluorescence test. ${ }^{23} \mathrm{It}$ is important to use a MOMP monoclonal as most EIA tests have a stage in which the LPS is solubilised. The introduction of integral blocking tests is to be welcomed and provides another opportunity for confirmation; preliminary experience with one such test (Chlamydiazyme Blocking Assay, Abbott Laboratories) is encouraging (Mumtaz and Ridgway, unpublished observations). Positive or borderline results which fail to confirm should be reported as equivocal, and a further specimen requested. ${ }^{23}$ False positive EIA results are particularly liable to occur with rectal specimens, ${ }^{24}$ which should not be tested by this technique.

Recent technological advances have resulted in membrane EIA tests. Coleman et al reported that TestPack Chlamydia (Abbott Laboratories) had a sensitivity of $76.5 \%$ and a specificity of $99.5 \%$ compared with culture or confirmed Chlamydiazyme EIA, or both. ${ }^{25}$ The prevalence of $C$ trachomatis was $11.6 \%$, giving a PVP of $96.2 \%$. These tests are simple to perform and their possible use by general practitioners and others who may have little understanding of the possible pitfalls is worrying. Caution is necessary when interpreting positive results in low risk populations, and the need to confirm such results in the manner mentioned above still applies.

\section{Nucleic acid probes}

Non-culture detection technology continues to advance. Mearns et al have reported the use of a sensitive immune dot blot test relying on a ${ }^{125} \mathrm{I}$ labelled, genus specific, monoclonal antibody directed against LPS. ${ }^{26}$ The test is convenient for the batch testing of samples, but requires three days to perform satisfactorily. DNA hybridisation methods have also been applied to chlamydial diagnosis. ${ }^{27}$ In general these tests are highly specific, but lack the sensitivity of the most sensitive methods. The need for radioactive probes limits their popularity, although the future use of non-radioactive ones, such as sulfonated DNA or biotinylated DNA, may solve this problem. Future use of DNA probes directed against ribosomal RNA rather than against single copy plasmid DNA, and the use of the Probe Assay Chemiluminescence Enhanced (PACE, Gen-Probe Inc) test, or the polymerase chain reaction (PCR) may improve sensitivity. The results of comparative field studies with these tests are awaited with interest. Problems are likely to remain, however, that of DNA contamination in the PCR being a case in point.

\section{Urine testing}

To obviate the need to pass a swab down the 
male urethra interest has been shown in testing a first catch urine sample for chlamydiae by non-culture methods. Testing of urine by cell culture has been dismissed in the past as too insensitive, ${ }^{28}$ but the advent of more sophisticated technology has led to a reassessment. Surprisingly, few peer reviewed reports have been published as yet, despite considerable clinical and commercial interest. Caul et al reported a preliminary study in a letter, but gave no details of the sensitivity and specificity of urine testing by EIA compared with their testing of urethral swabs by direct immunofluorescence. ${ }^{29}$ They particularly advocated the use of urine testing in asymptomatic - that islow risk men. In a follow up letter Matthews and Wise reported a sensitivity of $76.5 \%$ for first catch urine testing by IDEIA compared with testing urethral swabs in cell culture. ${ }^{30}$ Details of specificity or of any confirmatory tests were not given. Paul and Caul compared the results of testing first catch urine specimens from a small group of patients by three EIAs. ${ }^{31}$ Positive results were confirmed by direct immunofluorescence examination (MOMP specific) of the centrifuged urine specimens. Of the 21 urine specimens positive by IDEIA, 19 were confirmed by direct immunofluorescence. The two EIA positive urine samples that were negative by direct immunofluorescence were from women who both had EIA positive cervical swabs. All 19 men whose urine samples were EIA positive had EIA positive urethral swabs. Although these early results are encouraging, it is clear that much further work is required before urine testing in men for chlamydial infection can be recommended confidently, and it is very unlikely that this approach will have a role in detecting the predominantly cervical infection of women.

\section{Antibody detection tests}

A serological test with a high PVP for chlamydial infection continues to be elusive. There is no doubt that improvements in technology have provided important epidemiological data, but the use of serology for diagnostic purposes remains limited. In common with the use of serology for the diagnosis of many infections, a change in the titre of antibody is the most reliable criterion, particularly the demonstration of a fourfold or greater rise in titre. The use of single high titres for diagnosis is generally unreliable.

In the complement fixation test genus specific LPS is used to measure antibody. The test is very insensitive, detecting only deepseated chlamydial infection, such as LGV, psittacosis/ornithosis, and possibly $C$ pneumoniae respiratory infection. Furthermore, the test does not permit differentiation between causative organisms. A single antibody titre of 32 or greater in the presence of a suggestive clinical picture is strong evidence for LGV. The differentiation between $C$ psittaci and $C$ pneumoniae respiratory infection is less clear cut. Furthermore, a positive complement fixation test is not always seen in $C$ pneumoniae infection, or may be delayed. ${ }^{32}$
The microimmunofluorescence (MIF) test of Wang and Grayston is predominantly species and serovar specific. ${ }^{33}$ It is technically demanding, however, and antigens are not yet readily available. The test can be used to detect either specific IgG or IgM. Various modifications to the test have been proposed and used, such as pooling of antigens, ${ }^{34}$ but these tests are generally less species specific. The whole inclusion immunofluorescence (WIF) test of Richmond and Caul utilises a cross reacting strain of Chlamydia spp (such as an LGV 2 strain). ${ }^{35}$ The test is sensitive, but only genus specific. It cannot therefore be used to differentiate between the species. One study showed that half of the antibody detected in serum samples by the WIF test was due to $C$ pneumoniae infection when compared with species specific microimmunofluorescence testing. ${ }^{36}$ This study also confirmed the findings of others, that chlamydial antibody is common in the general population. Hagay et al compared a whole inclusion immunoperoxidase test for chlamydial IgG and IgA with cell culture for the diagnosis of urethritis in men. ${ }^{37}$ The results did not establish serology as a useful procedure in this setting. Thus an IgG titre of $>64$ gave a PVP of $67 \%$, and a PVN of $79 \%$ and an IgA titre of $>16$ a PVP of $74 \%$ and a PVN of $86 \%$, compared with culture: the prevalence of chlamydial infection by culture was $33 \%$. Several EIA tests have been described for antibody detection, again utilising cross reacting chlamydial antigen (usually LGV serovars). These tests are also genus specific. Contrary to the impression given by the manufacturers of a number of commercially available products, they cannot diagnose $C$ trachomatis infections specifically, and are not at all specific for the diagnosis of acute chlamydial infections.

The detection of specific IgM antibody in neonatal pneumonitis is strong evidence of a chlamydial aetiology. $C$ pneumoniae is difficult to isolate routinely which means that serology is the only practical method. Differentiation from $C$ psittaci infection may be very difficult as all readily available tests are genus specific. The complement fixation test is likely to be higher in psittacosis/ornithosis than in $C$ pneumoniae infection, but delay in appearance of antibody to the latter may obscure a serological diagnosis.

Southgate et al detected local cervical antibody by microimmunofluorescence in a low risk group (isolation rate of $8 \%$ ). ${ }^{38}$ They reported a PVP of $67 \%$ for local IgG compared with culture, a result which questions the advisability of using such an approach to detect infection in low risk groups.

\section{Discussion}

There remains a need for chlamydial diagnostic tests of high sensitivity and specificity which are easy to use and also cost effective. Advances in techniques which may ultimately achieve this goal continue. For the present, the need for a chlamydial diagnostic service is obvious, but enthusiasm must be balanced against the 
problems and limitations of currently available tests and their interpretation. Laboratory staff need to assess carefully the level of service required, the level of expertise available to them, and the economics of testing. Close liaison with clinicians is essential. Specimen collection must be monitored, particularly with regard to quality. The routine use of tests that have not been evaluated adequately is unsound, as is the use of such tests on specimens for which they were not designed, or where reliability has not been proved. Deviations from the manufacturer's written instructions should be carefully evaluated before clinical decisions are taken on the basis of the results. Positive antigen tests unsupported by a confirmatory test would be unacceptable in many other areas of microbiology, and should be avoided because a false positive diagnosis of sexually transmitted chlamydial infection can have a profound effect on the social life of a patient. Furthermore, confirmation is particularly important where litigation is under consideration, for example, in suspected cases of rape or child abuse. For the present, attempted culture should always be undertaken in such cases. ${ }^{39}$ Routine antigen detection tests in low risk populations should be used primarily to exclude chlamydial infection. Positive results should be confirmed and preferably repeated using a different method. Routine tests of cure are a waste of valuable resources because they are rarely positive and may cause confusion owing to the detection of non-viable chlamydial antigen. ${ }^{40}$ Generally, antibody investigations have little role in the routine diagnosis of genital chlamydial infections, and are at best complementary to antigen detection or culture.

1 Grayston JT, Wang S-P, Kuo C-C, Campbell LA. Current knowledge on Chlamydia pneumoniae, strain TWAR, an important cause of pneumonia and other acute respiratory important cause of pneumonia and other acute respiratory

2 Mårdh P-A, Paavonen J, Puolakkainen M. Chlamydia. New York: Plenum Medical, 1989.

3 Mårdh P-A, Zeeberg B. The toxic effect of sampling swabs and transportation test tubes on the formation of intracytoplasmic inclusions of Chlamydia trachomatis. $B r$ $J$ Vener Dis 1981;57:268-72.

4 Mahony JB, Chernesky MA. Effect of swab type and storage temperature on the isolation of Chlamydia trachomatis from clinical specimens. J Clin Microbiol 1985;22:865-7.

5 Barnes RC. Laboratory diagnosis of human chlamydial infections. Clin Microbiol Rev 1989;2:119-36.

6 Moncada J, Schachter J, Shipp M, Bolan G, Wilber J. Cytobrush in collection of cervical specimens for detection of Chlamydia trachomatis. J Clin Microbiol 1989;27: 1863-6

7 Taylor-Robinson D. Detecting Chlamydia trachomatis by direct immunofluorescence using a Cytobrush sampling technique. Genitourin Med 1989;65:130.

8 Ciotti RA, Sondheimer SJ, Nachamkin I. Detecting Chlamydia trachomatis by direct immunofluorescence using a Cytobrush technique. Genitourin Med 1988;64:245-6.

9 Goh BT, Dunlop EMC, Darougar S, Woodland R. Three sequential methods of collecting material from the urethra of men for culture for Chlamydia trachomatis. Sex Transm of men for culture for $1985 ; 12: 173-6$.

10 Dunlop EMC, Goh BT, Darougar S, Woodland R. Triple culture test for diagnosis of chlamydial infections of the culture test for diagnosis of chlamydial infections of
female genital tract. Sex Transm Dis 1985;12:68-71.

11 Munday PE, Carder JM, Hanna NF, Taylor-Robinson D. Is one swab enough to detect chlamydial infection of the cervix? Br J Vener Dis 1984;60:384-6.

12 Manuel ARG, Veeravahu M, Matthews RS, Clay JC. Pooled specimens for Chlamydia trachomatis: new approach to increase yield and cost efficiency. Genitourin Med 1987;63:172-5.

13 Lefebvre J, Laperiere H, Rousseau H, Masse R. Comparison of three techniques for detection of Chlamydia trachomatis in endocervical specimens from asymptomatic women. J Clin Microbiol 1988;26:726-31.

14 Mumtaz G, Ridgway GL, Nayagam A, Oriel JD. Enzyme immunoassay compared with cell culture and immunofluorescence for detecting genital chlamydia. J Clin Pathol 1989;42:658-60.

15 Forster GE, Cookey I, Munday PE, et al. Investigation into the value of Papanicolaou stained cervical smears for the diagnosis of chlamydial cervical infections. J Clin Pathol 1985;38:399-402.

16 Tam MR, Stephens RS, Kuo C-C, Holmes KK, Stamm WE, Nowinski RC. Use of monoclonal antibodies to Chlamydia trachomatis as immunodiagnostic reagents.
In: Mărdh P-A, Holmes $\mathrm{KK}$, Oriel JD, et al, eds. Chlamydial infections. Amsterdam: Elsevier Biomedical Press, 1982:317-20.

17 Thomas BJ, Evans RT, Hawkins DA, Taylor-Robinson D Sensitivity of detecting Chlamydia trachomatis elementary bodies in smears by use of a fluorescein labelled monoclonal antibody: comparison with conventional chlamydial isolation. J Clin Pathol 1984;37:812-6.

18 Jones BR, Taylor-Robinson D. Observations on and future trends in chlamydial research. Br Med Bull 1983;39. 201-3.

19 Mumtaz G, Mellars BJ, Ridgway GL, Oriel JD. Enzyme immunoassay for the detection of Chlamydia trachomatis antigen in urethral and endocervical swabs. J Clin Patho 1985;38:740-2.

20 Taylor-Robinson D, Thomas BJ, Osborn MF. Evaluation of enzyme immunoassay (Chlamydiazyme) for detecting Chlamydia trachomatis in genital tract specimens. J Clin Pathol 1987;40:194-9.

21 Thomas BJ, Osborn MF, Gilchrist C, Taylor-Robinson D. Improved sensitivity of an enzyme immunoassay IDEIA for detecting Chlamydia trachomatis.J Clin Pathol 1989;42:759-62.

22 Saikku P, Puolakkainen M, Leinonen $M$, Nurminen $M$ Nissinen A. Cross-reactivity between Chlamydiazyme and Acinetobacter strains. N Engl J Med 1986;314:922.

23 Mumtaz G, Ridgway G, Oriel JD. Chlamydia detection in extrogenital specimens. Lancet 1986;ii: 1156.

24 Rothburn MM, Mallinson H, Mutton KJ. False positive ELISA for Chlamydia trachomatis recognised by atypical morphology on fluorescent staining. Lancet 1986;ii: 982-3.

25 Coleman P, Varitek IK, Mushahlewar IK, et al. TestPack Chlamydia, a new rapid assay for the direct detection of Chlamydia trachomatis. J Clin Microbiol 1989;27:2811-4.

26 Mearns G, Richmond SJ, Storey CC. Sensitive immune dot blot test for diagnosis of Chlamydia trachomatis infection. $J$ Clin Microbiol 1988;26:1810-3.

27 Hyypiä T, Jalava A, Larsen SH, Terho P, Hukkanen V. Detection of Chlamydia trachomatis in clinical specimens by nucleic acid spot hybridisation. J Gen Microbiol 1985;131:975-8.

28 Smith TF, Weed LA. Comparison of urethral swabs, urine and urinary sediment for the isolation of Chlamydia. $J$ Clin Microbiol 1975;2:134-5.

29 Caul EO, Paul ID, Milne JD, Crowley T. Non-invasive sampling method for detecting Chlamydia trachomatis. Lancet 1988;ii:1246-7.

30 Matthews RS, Wise R. Non-invasive sampling method for detecting Chlamydia trachomatis. Lancet 1989;i:96-7.

31 Paul ID, Caul EO. Evaluation of three Chlamydia trachomatis immunoassays with an unbiased, noninvasive clinical sample. J Clin Microbiol 1990;28:220-2.

32 Grayston JT, Kuo C-C, Wang S-P, Altman J. A new Chlamydia psittaci strain called TWAR from acute respiratory infections. $N$ Engl J Med 1986;315:161-8.

33 Wang S-P, Grayston JT. Immunologic relationship between genital TRIC and lymphogranuloma venereum and related organisms in a new microtitre indirect immunofluorescence test. Am J Ophthalmol 1970;70:367-74.

34 Treharne JD, Darougar S, Jones RB. Modifications of the micro-IF test to provide a routine serodiagnostic test for chlamydial infection. J Clin Pathol 1977;30:510-7.

35 Richmond SJ, Caul EO. Fluorescent antibody studies in chlamydial infections. J Clin Microbiol 1975;1:345-52.

36 Forsey T, Stainsby K, Hoger PH, Ridgway GL, Darougar $\mathrm{S}$, Fischer-Brugge U. Comparison of two immunofluorescence tests for detecting antibodies to Chlamydia trachomatis. Eur J Epidemiol 1986;2:163-4.

37 Hagay ZJ, Sarov B, Sachs J, Shaked O, Sarov I. Detecting Chlamydia trachomatis in men with urethritis: serology v.
isolation in cell culture. Genitourin Med 1989;65:166-70.

38 Southgate LJ, Treharne JD, Forsey T. Chlamydia trachomatis and Neisseria gonorrhoeae infections in trachomatis and Neisseria gonorrhoeae infections in
women attending inner city general practices. $\mathrm{Br} \mathrm{Med} \mathrm{J}$ women attending

39 Hammerschlag MR, Rettig PJ, Shields ME. False positive results with the use of chlamydial antigen detection tests in the evaluation of suspected sexual abuse in children. Paediatr Inf Dis 1988;7:11-4.

40 Nachamkin I, Sawyer K, Skalina D, Crooks GW, Ciotti R, Sondheimer SJ. Test-of-cure analysis by direct immunofluorescence for Chlamydia trachomatis after antimicrobial therapy. J Clin Microbiol 1987;25:1774-5. 